4. Шор Н. 3., Журбенко Н. Г. Метод минимизации, использующий операцию растяжения пространства в направлении разности двух последовательных градиентов. Кибернетика. 1971. № 3. С. 51-59.

5. Лаптин Ю. П., Бардадым Т. А. Проблемы определения коэффициентов точных штрафных функций. Кибернетика и системный анализ. 2019.

6. Нурминский Е. А. Проекция на внешне заданные полиэдры. Вычисл. матем. и матем. физ. 2008. Т. 48. № 3. С. 387-396.

7. Стецюк П. И. Программа ralgb5 для минимизации овражных выпуклых функций. Математичне та програмне забезпечення інтелектуальних систем. 2016. С. 185-197.

\title{
ON APPROXIMATE CALCULATION OF THE COEFFICIENTS OF EXACT PENALTY FUNCTIONS
}

Simplified procedures to specify the penalty coefficients more exactly are proposed. The results of computational experiments on randomly generated linear programming problems are given.

Key words: exact penalty functions, structured optimization problems, decomposition methods.

Получено 30.01.2019

УДК 519.9

DOI: $10.32626 / 2308-5878.2019-19.60-64$

О. М. Литвин*, д-р фіз.-мат. наук,

О. О. Литвин*, д-р фіз.-мат. наук,

О. В. Ткаченко**, канд. фіз.-мат. наук

*Українська інженерно-педагогічна академія м. Харків, **ДП «Івченко-Прогрес», м. Запоріжжя

\section{МЕТОД ОДНОЧАСНОГО РІВНОМІРНОГО НАБЛИЖЕННЯ СПЛАЙНАМИ ТРИГОНОМЕТРИЧНИХ ФУНКЦІЙ ТА ЇХ ПОХІДНИХ}

Наведені теореми про найкраще наближення сплайнами тригонометричних функцій та їх похідних, з дотриманням ізогеометричних властивостей.

Ключові слова: найкраще наближення сплайнами, розривні періодичні сплайни.

Вступ. На даний час наближення функцій із збереженням ізогеометричних властивостей досліджувалося в багатьох працях Відмітимо, зокрема, роботи [1-5] присвячені наближенню функцій і збереження ізогеометричних властивостей. Використовуються також узагальнені сплайн-функції. У роботах $[4,5]$ ізогеометричні власти- 
вості забезпечувалися автоматично формулами інтерлінації із автоматичним збереженням потрібного класу диференційовності.

Зупинимось детальніше на використанні узагальнених сплайнів для наближення функції однієї змінної із збереженням іiі ізогеометричних властивостей. Як відомо [1], якщо у вузлах сітки $\Delta: a=x_{0}<x_{1}<$ $<\ldots<x_{n}=b$ задані значення функції $y=f(x): y_{i}=f\left(x_{i}\right)(i=\overline{0, n})$, то кубічним інтерполяційним сплайном на сітці $\Delta$ називається функція $S_{3}(f, x)$, неперервна на $[a, b]$ разом із своєю першою та другою похідними, яка $є$ кубічним поліномом на кожному 3 відрізків $\left[x_{0}, x_{1}\right]$, $\left[x_{1}, x_{2}\right], \ldots,\left[x_{n-1}, x_{n}\right]$ і задовольняє умови $S_{3}\left(f, x_{i}\right)=y_{i}, i=\overline{0, n}$.

Доведено, що такий сплайн єдиний. Наведемо алгоритм його чисельної побудови. Позначимо $S_{3}{ }^{\prime \prime}\left(f, x_{i}\right)=M_{i}, i=\overline{0, n}$. Побудуємо сплайн на відрізку $\left[x_{i}, x_{i+1}\right], i=\overline{0, n-1}$. Оскільки на цьому відрізку $S_{3}(f, x)-$ це поліном третього степеня, то його друга похідна $є$ поліномом першого степеня, тобто це лінійна функція, графік якої проходить через точки $\left(x_{i-1}, M_{i-1}\right)$ i $\left(x_{i}, M_{i}\right),(i=1, n)$. Користуючись формулою для рівняння прямої, яка проходить через дві задані точки, можна написати

$$
S_{3}^{\prime \prime}(f, x)=M_{i-1} \frac{x_{i}-x}{h_{i}}+M_{i} \frac{x-x_{i-1}}{h_{i}}, h_{i}=x_{i}-x_{i-1} .
$$

Проінтегруємо двічі рівність (1) i, знайшовши невідомі довільні сталі, що виникають під час інтегрування на відрізку $\left[x_{i-1}, x_{i}\right]$, отримуємо:

або

$$
S^{\prime}(f, a)=f_{a}{ }^{\prime}, S^{\prime}(f, b)=f^{\prime}{ }_{b}
$$

$$
S_{3}{ }^{\prime \prime}(f, a)=f_{a}^{\prime \prime}, S_{3}{ }^{\prime \prime}(f, b)=f_{b}^{\prime \prime}
$$

Кубічний сплайн на кожному проміжку $\left[x_{i}, x_{i+1}\right]$ сітки $є$ кубічним поліномом, який може бути представлений через значення наближуваної функції і другі похідні на кінцях цього проміжку [a, b]

$$
S_{3}(f, x)=\sigma(x)+\varphi(1-t) h_{i}^{2} M_{i}+\varphi(t) h_{i}^{2} M_{i+1},
$$

де

$$
\sigma(x)=(1-t) f_{i}+t f_{i+1}, \varphi(t)=\frac{t^{3}-t}{6}, t=\frac{x-x_{i}}{h_{i}}, M_{i}=S^{\prime \prime}\left(x_{i}\right) .
$$

Невідомі вузлові значення $M_{i}$ других похідних знаходяться шляхом розв'язання системи лінійних алгебраїчних рівнянь, яка отримується з умови гладкості сплайна $S_{3}(f, x)$, відповідних умовам гладкості сплайна класу $S_{3}(f, x) \in C^{2}[a, b]$. Відомо [1-3], що у пове- 
дінці класичного кубичного сплайна виникають проблеми внаслідок використання функцій у формулі $\varphi(t)$ вище написаної. Тому в [1-3], використовується функція $\varphi(p, t) \in C^{2}[0,1]$, яка має вільний параметр $p$ для управління поведінкою сплайна $S_{3}(f, x)$ і при $p=0$ співпадає з $\varphi(t)=\frac{\left(t^{3}-t\right)}{6}$, що породжує класичний кубічний сплайн.

У роботі пропонується доповнення до методу інтерлінації з автоматичним збереженням ізогеометричних властивостей (монотонного спадання, зростання, опуклості тощо), який досліджується детальніше лише для тригонометричних функцій $\sin 2 \pi t, \cos 2 \pi t, 0 \leq t \leq 1$.

Наведені основні твердження методу, основані на використанні основної теореми про найкраще наближення монотонної на інтервалі $[a, b]$ неперервної функції $f(x) \in C[a, b]$ сталою.

Крім того, якщо ми наближуємо $f(x)$ кусково-сталими сплайнами найкращого наближення в нормі $C[a, b]$, то інтеграл від такого сплайна буде сплайном 1-го степеня, який теж дасть найкраще наближення для інтеграла. Тобто наближуючи невідому $f$ " $(x)$ кусково-сталими сплайнами 3 невідомими значеннями сталих на підінтервалах монотонності, після інтегрування ми отримаємо сплайн 1-го степеня, який найкраще наближує $f(x)$ на $[a, b]$. Далі, інтегруючи цей сплайн 1-го степеня, отримаємо сплайн 2-го степеня, у якому можна вибрати невідомі сталі так, що цей сплайн буде найкраще наближувати $f(x)=\int f^{\prime}(x) d x+C$.

Наведено аналіз результатів обчислювального експерименту, який підтверджує ефективність запропонованого методу одночасного наближення похідних і функції у припущенні, що нам відомі значення $y_{k}=f\left(x_{k}\right), k=\overline{1, Q}$, а також інтервали монотонності $f^{\prime}(x)$ або $f^{\prime \prime}(x)$.

Означення. Кусково-сталий сплайн [6]

$$
S(r, m, t)=\mid \begin{aligned}
& \frac{i}{m}, \text { if } t=\frac{1}{2 \pi} \arcsin \frac{i}{m}, \quad i=\overline{0, m}, \\
& \frac{i+0,5}{m}, \text { if } T_{i}<t<T_{i+1}, \quad i=\overline{0, m-1} .
\end{aligned}
$$

Теорема 1. Сплайн $S(-1, n, t)$ будемо називати кусково-сталим сплайном (сплайном степеня 0), який найкраще наближує функцію $y=\sin 2 \pi t$ на інтервалі $0 \leq t \leq 0,25$.

Доведення цієї теореми базується на відомому твердженні: найкраще наближення монотонної на $[a, b]$ функції $f(x)$ сталою $C$ в нормі $C[a, b]$ існує і єдине, і визначається формулою 


$$
C=\frac{\max _{a \leq x \leq b} f(x)+\min _{a \leq x \leq b} f(x)}{2}=\frac{f(a)+f(b)}{2} .
$$

При цьому похибка наближення

$$
\max |f(x)-C| \leq \frac{M-m 0}{2},
$$

де $m 0=\min f(x), M=\max f(x)$.

$$
a \leq x \leq b \quad a \leq x \leq b
$$

3 теореми 1 витікає, що для зменшення похибки наближення в $m$ разів достатньо розбити інтервал монотонності $[a, b]$ на $m$ підінтервалів $\left[x_{i}, x_{i+1}\right], i=\overline{0, m-1}, x_{0}=a, x_{m}=b, x_{i}=a+\frac{b-a}{m} i$ і в кожному 3 цих підінтервалів наближувати $f(x)$ сталою $C_{i}=\frac{f\left(x_{i}\right)+f\left(x_{i+1}\right)}{2}$. Тоді результуючий сплайн кусково-сталий буде наближувати $f(x)$ в нормі $C[a, b]$ з похибкою $\varepsilon=\frac{|f(b)-f(a)|}{2 m}$. Зауважимо, що точки $x_{i}$ цього розбиття знаходяться як розв'язки рівнянь $f(x)=f(a)-\frac{f(b)-f(a)}{m} i, \quad i=\overline{0, m}, \quad$ якщо $\quad f(a)<f(b) \quad$ і $f(x)=f(a)-\frac{f(a)-f(b)}{m} i, i=\overline{0, m-1}$, якщо $f(a)>f(b)$.

Теорема 2. Сплайн 1-го степеня $S(0, m, t) \in C[0,0.25]$, який найкраще наближує $\int f(t) d t+C=-\frac{\cos 2 \pi t}{2 \pi}+C$, можна представити у вигляді

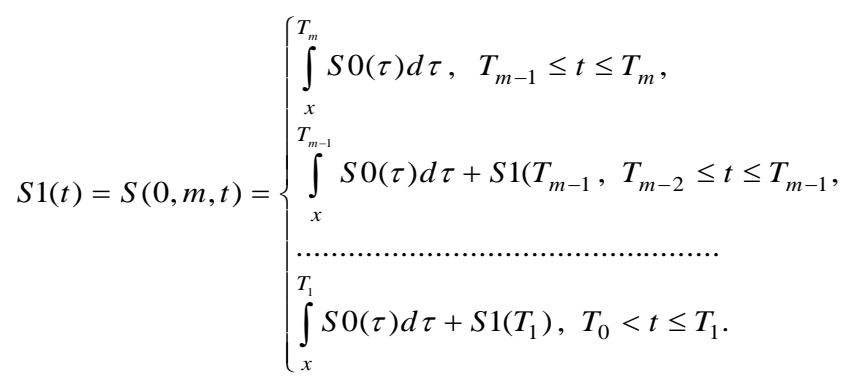

Таким чином, з формули (8) випливає, що $S 1\left(T_{m}\right)=0$, що означає автоматичне виконання відомого співвідношення

$$
\left(-\frac{\cos 2 \pi t}{2 \pi}\right)_{t=0,25}=-\frac{\cos \frac{\pi}{2}}{2 \pi}=0 .
$$


На рисунку показано графік функції $y=S 1(t)$ сплайна 1 степеня, $t \in[0,1]$, який отримується шляхом інтегрування кусково-сталого сплайна найкращого наближення функції $y^{\prime}(t)=\sin 2 \pi t, t \in[0,1]$.

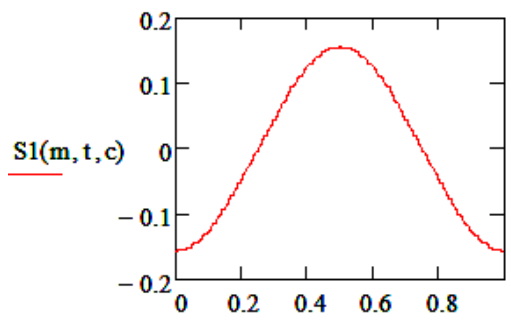

Рисунок.

Висновки. Наведені твердження, які описують метод одночасного рівномірного наближення сплайнами тригонометричних функцій та їх похідних. Показано графік функції, яка отримується шляхом інтегрування кусково-сталого сплайна ії похідної.

\section{Список використаних джерел:}

1. Квасов Б. И. Методы изогеометрической аппроксимации сплайнами. М. : ФИЗМАТЛИТ, 2006.

2. Spath H. Spline Algorithms for Curves and Surfaces. Winnipeg: Uni-tas Mathematica Publ. 1974.

3. Богданов В. В., Волков Ю. С. Выбор параметров обобщённых кубических сплайнов при выпуклой интерполяции. Сиб. журн. вычисл. математики. Новосибирск, 2006. Т. 9. № 1. С. 5-22.

4. Литвин О.М. Інтерлінація функцій та деякі іï застосування. Харків : Основа, 2002. $544 \mathrm{c}$.

5. Сергиенко И. В., Литвин О. Н., Литвин О. О., Ткаченко А. В., Грицай О. Л. Интерлинация эрмитового типа на системе непересекающихся линий (Обзор). Кибернетика и системный анализ. 2015. Т. 51, № 2. С. 134-144.

6. Литвин О. М. Періодичні сплайни і новий метод розв'язання плоскої задачі рентгенівської комп'ютерної томографії. Системний аналіз, управління і інформаційні технології: Вісн. Харків. держ. політех. ун-ту : зб. наук. праць. 2000. № 125. С. 27-35.

\section{BY THE METHOD OF SIMULTANEOUS UNIFORM APPROXIMATION BY SPLINES OF TRIGONOMETRIC FUNCTIONS AND THEIR DERIVATIVES}

Method is presented on the best approximation by splines of trigonometric functions and their derivatives, with respect to isogeometric properties.

Key words: best approximation by splines, discontinuous periodic splines.

Одержано 05.02.2019 